Review Article

\title{
Worthwhile Relevance of Infrared Spectroscopy in Characterization of Samples and Concept of Infrared Spectroscopy-Based Synchrotron Radiation
}

\author{
Ayodele Temidayo Odularu \\ Department of Chemistry, University of Fort Hare, Private Bag X1314, Alice 5700, South Africa \\ Correspondence should be addressed to Ayodele Temidayo Odularu; 201106223@ufh.ac.za
}

Received 6 April 2020; Revised 22 August 2020; Accepted 26 August 2020; Published 31 August 2020

Academic Editor: Renata Diniz

Copyright ( $\odot 2020$ Ayodele Temidayo Odularu. This is an open access article distributed under the Creative Commons Attribution License, which permits unrestricted use, distribution, and reproduction in any medium, provided the original work is properly cited.

\begin{abstract}
The study explores the nitty-gritty of infrared spectroscopy. Firstly, the review gives a concise history of infrared discovery and its location in the electromagnetic spectrum. Secondly, the infrared spectroscopy is reported for its mechanism, principles, sample preparation, and application for absence and presence of functional groups determination in both ligands and coordination compounds. Thirdly, it helps in purity determination of unknown samples. Additional studies regarding this study entail infrared spectroscopy-based synchrotron radiation. It serves as a giant microscope to give detailed information of samples under investigation compared to the conventional infrared instrument. Infrared will continue to be useful to both chemical and pharmaceutical industries, in order to make chemical products and manufactured drugs put on wholesome integrity.
\end{abstract}

\section{Introduction}

Infrared is a convenient tool relevant to chemistry and chemistry related disciplines (inorganic and organic chemistry and pharmacy) $[1,2]$. Reports of its relevancy could be seen in studies indicating the detection of absence and presence of functional groups in ligands and evidence of coordination in the proposed compounds [3]. Infrared has versatile applications to differentiate between the hydroxyl and amine groups from the spectra [4] and determine the presence of water in case of no thermal analyzer [5], and it can be used for purity determination $[5,6]$ in case of no elemental analyzer. The study aimed to provide the history, pragmatic uses, maintenance of infrared spectroscopy, and concise infrared spectroscopy based synchrotron radiation.

\section{Electromagnetic Spectrum and Spectroscopy}

The electromagnetic spectrum is made up of an array of regions useful to chemists and the scientific world [7]. It is also a range of frequencies of electromagnetic radiation and their corresponding wavelengths with photon energies [8] as shown in Figure 1. The range has electromagnetic waves with minimum frequencies of below one g hertz to a maximum of over $10^{28}$ hertz, equivalent to wavelengths [9] or from a fraction of an atomic nucleus size to a thousand kilometre [8]. This frequency range is divided into distinct regions and the electronic waves within every frequency region [10]. They are named differently starting from the low frequency (long wavelength) to the spectrum end [11]. The spectrum entails radio waves, microwaves, infrared, visible light, ultraviolet, X-rays, and gamma rays at the high frequency (short wavelength) as shown in Figure 1. The electromagnetic waves in each of the regions possess various characteristics, such as mode of production, mode of interaction with matter, and their pragmatic applications [12].

2.1. Spectroscopy. A characterization technique known as spectroscopy could be used physically to separate electromagnetic waves of various frequencies $[13,14]$. Therefore, it generates a spectrum which shows the constituents' 


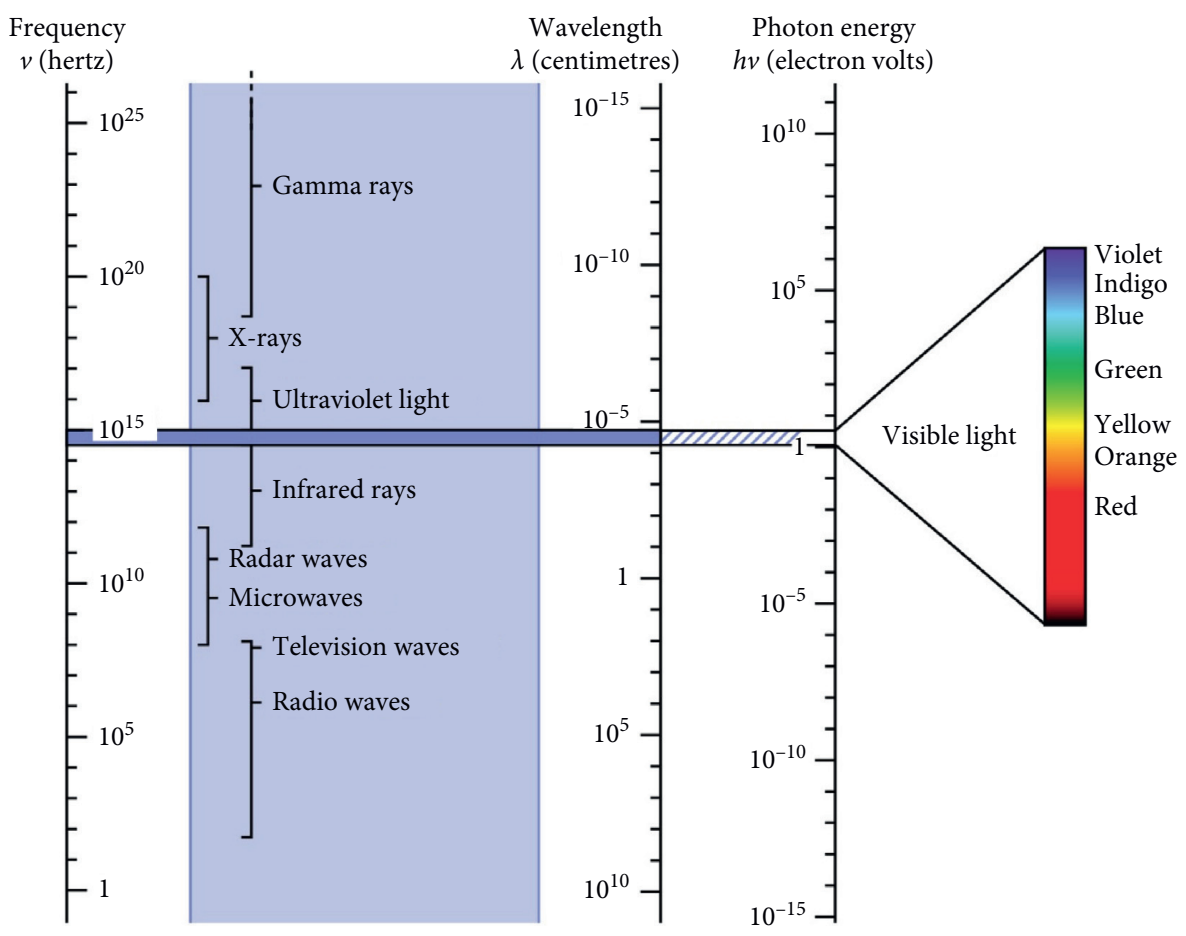

Figure 1: The electromagnetic spectrum.

frequencies $[15,16]$. Spectroscopy is used to study the interactions of the electromagnetic waves with matter $[17,18]$.

\section{Infrared}

\subsection{Infrared Spectrum and Spectroscopy (IR Spectroscopy)}

3.1.1. Infrared (IR) Spectrum. The infrared region of the electromagnetic spectrum is in three portions, namely, the near-, mid-, and far-IR [3, 19]. They are named based on their relationships with the visible spectrum [20]. The nearIR has a high energy of approximately $14000-4000 \mathrm{~cm}^{-1}$, which can excite harmonic vibrations [3]. The mid-IR has approximately $4000-400 \mathrm{~cm}^{-1}$ while the far-IR has approximately a low energy of $400-10 \mathrm{~cm}^{-1}$, which is next to the radar and microwave regions (Figure 1), and could be used for rotational spectroscopy [3].

3.1.2. Infrared (IR) Spectroscopy. Infrared spectroscopy also referred to as IR spectroscopy deals with the interaction of absorbed radiant energy in the infrared region of the electromagnetic spectrum with matter [21]. Infrared radiation has a longer wavelength and low frequency. The IR radiation is centered on spectroscopy [22]. It is used to identify chemical compounds with a current instrument called Fourier Transform Infrared (FTIR) spectrometer, which implements the principle of IR spectroscopy $[19,23,24]$. The FTIR is also a technique which measures and records infrared spectra [19].

In summary, IR spectroscopy uses IR spectrum to probe matter.
3.2. Mechanism of Action and Principles of Infrared Spectroscopy. The mechanism of action and principles of infrared spectroscopy complement each other $[25,26]$ as shown in Figure 2.

3.2.1. Infrared Mechanism of Action. Inside the infrared light source is an apparatus called interferometer. It guides the IR sample. The interferometer consists of a moving mirror which changes the IR light distribution when it passes through the sample $[27,28]$. The resulting signal called, "interferogram," is recorded directly, signifying the light output as a function of mirror position [29].

A data-processing technique called "Fourier transform" transforms the raw data into the expected results of the sample's spectrum $[24,30]$. Light output is a function of infrared wavelength. The sample's spectrum is mostly compared with a reference spectrum [Figure 2] in order to remove fixed-pattern noise [31]. The IR spectrometer having both reference and sample spectra are referred to as "a twobeam absorption spectrometer" [31].

3.3. Principles of IR Spectroscopy. The principle involves a process whereby a beam of IR light from IR source (Globar) is passed through a sample and the infrared spectrum is recorded $[22,24]$. The process is directed with the help of a monochromator (monochromatic beam of light), which is placed after IR source and before the IR detector, which interchanges in wavelength over time [32]. The sample absorbs light when there is resonant frequency [33]. Resonant frequency is when the IR frequency is the same as the vibrational frequency of the sample's bond [34-36]. The 


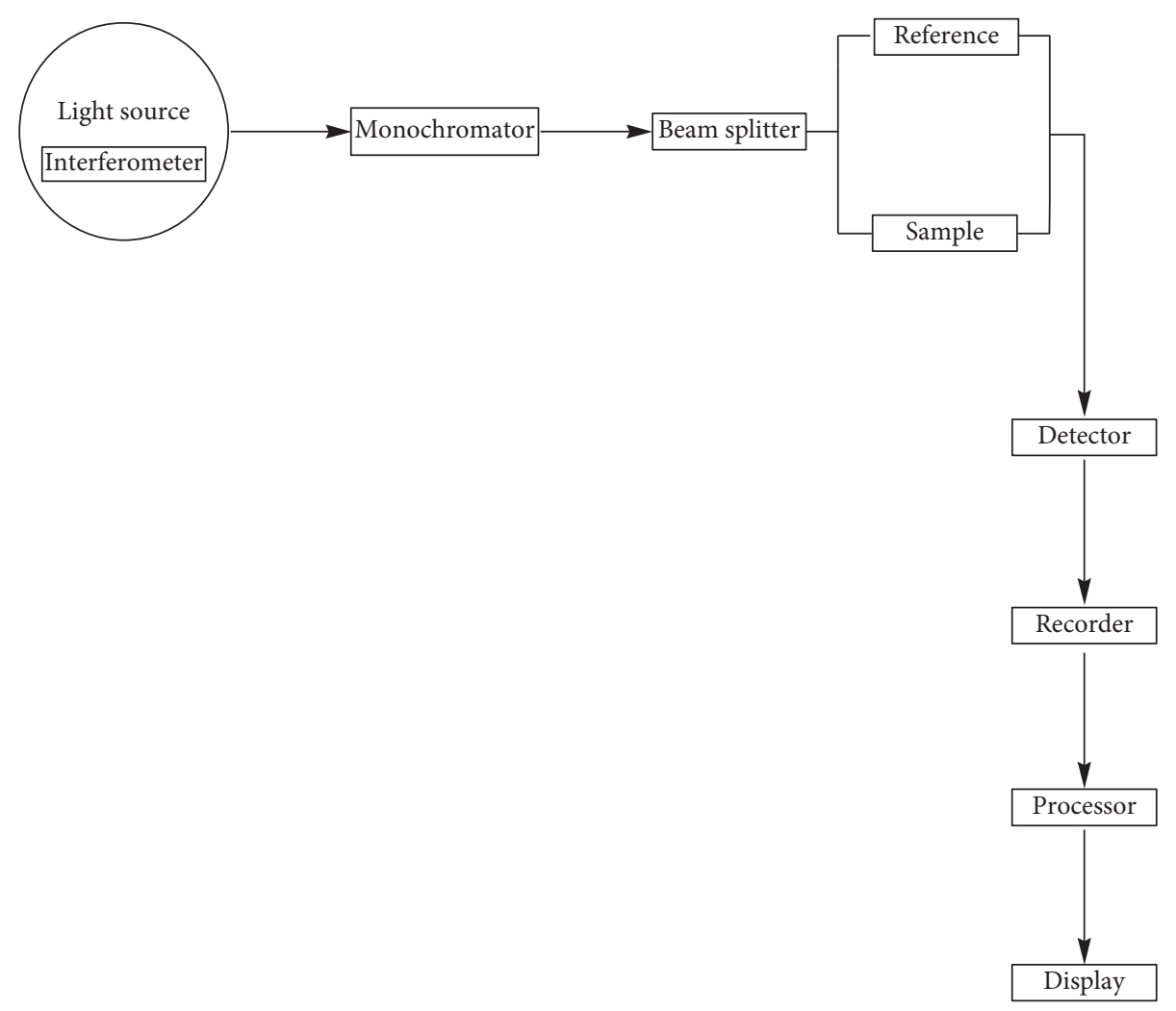

FIgURE 2: Schematic diagram of IR spectrometer with a two-beam absorption.

transmitted light shows the quantity of energy absorbed at every wavelength [37]. The Fourier transform instrument measures the entire wavelength at once and produces an absorbance or transmittance spectrum revealing the IR wavelengths, which the sample absorbs (Figure 2) [24]. Analysis of the spectrum gives details of the sample's molecular structure. This technique has been used on samples with covalent bonds; high levels of purity few IR active and complex molecular structures [38].

3.4. Theory of Infrared Spectroscopy. The IR spectroscopy is centered on the fact that molecules absorb specific frequencies which are characteristics of their chemical structures [22]. Hence, the resonant frequencies are related not only to the bonds but also to the atoms [39]. In other words, vibrational frequency could be connected with a particular bond type [40].

3.4.1. Vibrational Modes. A vibrational mode, also called vibrational degree of freedom, deals with ways where a molecule undergoes vibrations [41, 42]. A vibration mode could be IR active in a molecule if changes in permanent dipole are fundamental for a vibrational mode in a molecule $[43,44]$.

Molecules, such as carbon (IV) oxide, have 3N-5 degrees of vibrational with $\mathrm{N}$ atoms in the molecules [44, 45]. Linear molecules, like simple diatomic molecules (e.g., $\mathrm{CO}_{2}$; $\mathrm{O}=\mathrm{C}=\mathrm{O}$ ), have solely one bond and solely one vibrational bond calculated from $3 \mathrm{~N}-5$ degrees of vibrational modes. On the other side, nonlinear molecules, such as water, have $3 \mathrm{~N}-6$ degrees of vibrational modes $[44,46]$. Here, water has 3 atoms; that is, $3 \times 3-6=3$ degrees of freedom. Symmetrical molecules, such as $\mathrm{O}_{2}$, are observed solely in the Raman spectrum, not in the IR spectrum unlike unsymmetrical diatomic molecules, such as $\mathrm{CO}_{2}$ in the IR spectrum [47]. In the case of complex molecules, they have several bonds and they are observed in the IR spectrum [35]. The atoms in methylene (groups, found usually in organic compounds vibrate in six various ways, viz, rocking, scissoring, symmetric and antisymmetric stretching, twisting, and wagging [48].

\section{Sample Preparation for IR Characterization}

Unlike liquid state nuclear magnetic resonance (NMR), which requires right solvent, the sample for IR characterization does not require a solvent to dissolve it.

4.1. Solid Samples. Solid samples for IR characterization are prepared in four various ways. Firstly, a quantity of the studied sample is ground with fine purified salt, usually potassium bromide $(\mathrm{KBr})$ in an agate mortar with pestle to form a mixture. The $\mathrm{KBr}$ is used to record the IR spectra because it has a larger transmission window (100\%) in the IR compound than sodium chloride. The agate mortar with pestle is used to prevent contamination [18]. Thin film of the mull produced is smeared onto salt plates and measured by the infrared spectrophotometer [44]. The mixture gotten is then mechanically pressed to form a translucent pellet which 
allows the beam of light of the spectrophotometer to pass through it [44]. The second method entails a mixture of sample and an oily mulling agent which is ground also in a mortar with a pestle to form a Nujol [44]. The third method is adopted for polymeric materials [44]. This method is called the "cast film" technique $[44,49]$. Here, the sample is first dissolved in a suitable, nonhygroscopic solvent [44]. A drop of the solution gotten from the sample dissolution in a suitable nonhygroscopic solvent is deposited on the surface of sodium chloride or sodium bromide cell [44]. Analysis is conducted directly on the film formed on the cell after evaporation of the solution to dryness [44]. It is important to take caution to ensure the film is thin (not too thick) for the light to pass through; otherwise light cannot pass through [44]. This technique is suitable for qualitative analysis [44]. The last method is using microtomy to cut a thin $(20-100 \mu \mathrm{m})$ film from a solid sample [44]. It is one of the most essential ways to analyze the integrity of a preserved solid, such as a failed plastic product [44].

4.2. Liquid Samples. Here, liquid samples are placed in between two plates of a salt, such as sodium chloride, potassium bromide, or calcium fluoride [44]. The plates are usually transparent to the infrared light so that they do not introduce any lines onto the spectra [44].

4.3. Gaseous Samples. Sample cells with a long path length are needed for gaseous samples in order to make up for the dilution [44]. The path length of the sample cell depends on the concentration of the compound [44]. A sample gas concentration down to several hundred $\mathrm{ppm}$ requires a simple glass tube with a length of $5 \mathrm{~cm}$ to $10 \mathrm{~cm}$ walled with an ending of infrared windows, while a White cell with a mirror guided with infrared light can measure sample gas concentration below ppm [44]. The White cells are available with optical path length ranging from $0.5 \mathrm{~m}$ up to 100 metres [44].

In summary, samples prepared for IR characterization can be in solid, liquid, or gaseous states.

4.4. Photoacoustic Spectroscopy. In this spectroscopy, the solid or liquid sample requires minimal sample treatment $[44,50]$. The sample is placed into the sample cup with an insertion of a sealed photoacoustic cell for the spectral measurement [44]. The sample may be one solid piece, powder, or basically in any form for the measurement [44]. It is important to note that spectra obtained from different sample preparation methods will look slightly different from each other [44]. Differences in the samples' physical states bring about slight differences in spectra because of different sample preparation methods [44].

\section{Applications of Infrared Spectroscopy}

Infrared spectroscopy, being a simple as well as a reliable characterization technique, is applicable to all aspects of organic and inorganic chemistry [51]. It is also useful in research institutes and industries [52]. The purpose of adopting this characterization technique is to determine quality control, dynamic measurement, and monitoring applications such as the long-term unattended measurement of $\mathrm{CO}_{2}$ concentrations in greenhouses, growth chambers by infrared gas analyzers, and forensic analysis [53]. Changes in the character of a certain bond are measured and assessed at a specific frequency over a period of time [11]. Infrared measurements up to 32 times per second could be achieved with modern research instruments, as well as with other techniques simultaneously [54-57]. This enables the observations of chemical reactions and processes to be faster and more accurate [54].

5.1. Microelectronics and Semiconductors Infrared. Infrared spectroscopy has been used successfully in the field of microelectronics, as well as semiconductors infrared, such as amorphous silicon, silicon arsenide, and other gallium and silicon compounds $[58,59]$. Nowadays, the durability of the instruments has made them useful for field trials $[23,55]$.

5.2. Isotope Effects. The different isotopes in a particular species may give fine details in infrared spectroscopy, obtainable with species possessing different isotopes [60-62]. For instance, the $\mathrm{O}-\mathrm{O}$ stretching frequency (in reciprocal centimetres) and the wave number of absorbance, $v$, can be calculated using the formula $v=1 / 2 \pi c \sqrt{ } k / \mu$, where $v$ is the wavenumber in $\mathrm{cm}^{-1}, k$ is the spring constant for the bond, $c$ is the speed of light in $\mathrm{ms}^{-1}$, and $\mu$ is the reduced mass of the A-B system as shown in the following:

$$
\mu=\left(m_{\mathrm{A}} m_{\mathrm{B}}\right) / m_{\mathrm{A}}+m_{\mathrm{B}} \text { ( } m \text { is the mass). }
$$

Presently, the effect of isotopes on vibration and decay dynamics was observed compared to before. Antisymmetric stretch mode decay of interstitial oxygen entails the symmetric stretch mode with strong isotope dependence in some substances, such as germanium and silicon.

\subsection{Dimensional Infrared}

5.3.1. Two-Dimensional Infrared. The two types of two-dimensional IR are linear and nonlinear.

5.3.2. Linear Two-Dimensional Infrared Spectroscopy. Linear two-dimensional infrared bond spectroscopy analysis entails the use of two-dimensional infrared bond analyses on infrared spectra $[63,64]$. This is done in order to increase the spectra of a disturbed sample for simpler spectral analysis and improved resolution $[65,66]$. The pulse sequence is used to get a two-dimensional Fourier Transform Infrared spectrum $[67,68]$.

5.3.3. Nonlinear Two-Dimensional Infrared Spectroscopy. Nonlinear two-dimensional infrared spectroscopy is the infrared version of correlations spectroscopy [69]. Femtosecond infrared laser pulses development came along with 
nonlinear two-dimensional infrared spectroscopy [70, 71]. The experiment entails the first set of probe pulses useful to the sample, followed by a delay time for system relaxation [71-73]. The usual duration for the delay time requires zero to some picoseconds, which could be influenced by tens of femtosecond revolutions. A probe pulse is applied subsequently which results in the emission of a signal from the sample. Graphically, the nonlinear two-dimensional infrared spectrum is a two-dimensional correlation plot of the frequency $\omega_{1}$ excited by the first pump pulses and frequency $\omega_{3}$ excited by the probe pulse after the delay time. This supports the coupling observation between different vibrational modes because of its extremely high time resolution [74]. It could also be used to observe molecular dynamics on a picoseconds timescale, though still mainly a new technique, but its knowledge is increasing for fundamental research [74, 75]. As said previously, infrared spectroscopy could be used with other characterization techniques, such as two-dimensional nuclear magnetic resonance (NMR) [76, 77]. The nonlinear two-dimensional infrared technique spreads the spectrum in two dimensions and supports the observation of cross peaks, consisting of information of coupling between different modes [78, 79].

\section{Difference between Nonlinear Two- Dimensional Infrared Spectroscopy and 2D Nuclear Magnetic Resonance}

The nonlinear two-dimensional infrared spectroscopy is different from 2D NMR due to its involvement in the excitation to overtones [35]. The excitation outcome gives an excited state absorption peak located below the diagonal and cross peaks [73]. In 2DNMR, the two techniques, COSY and NOESY, are used often [80]. The cross peaks in the first are relevant to the scalar coupling, whereas, in the later, they are relevant to the spin transfer between diverse nuclei $[81,82]$. The 2DNMR techniques analogs are imitated in nonlinear two-dimensional IR spectroscopy, where the nonlinear two-dimensional IR spectroscopy with zero delay time corresponds to COSY [83]. In addition, nonlinear two-dimensional IR spectroscopy with fixed delay time permitting vibrational population transfer which corresponds to NOESY [84]. The COSY alternative of nonlinear two-dimensional IR spectroscopy has been used to determine the secondary structure of proteins [85].

\section{Purity Determination}

The challenge of values higher than $0.5 \%$ obtained from samples analyzed with elemental analyzer is giving a chance to infrared to be considered for purity determination of samples $[86,87]$. The application of the infrared technique to analyze samples for purity determination could be observed in three different ways, namely, old way, new way, and lack of reference [24, 88-90].

7.1. The Old Way. In the old way, the infrared spectrum of the unknown sample is taken occasionally and compared with the infrared spectrum of the known pure sample (reference) [91]. Any irrelevant peak suggests the presence of impurities [92]. If the peaks matched those of known impurities, they could apply the intensities to evaluate sample purity.

7.2. The New Way. The introduction of computers and Fourier Transform technology probably led to the use of differential spectroscopy in many industries [24]. Here, an unknown sample spectrum is also obtained occasionally and a stored spectrum of the known pure sample is subtracted digitally from it [24]. It leaves spectrum impurities, which could be compared with impurities' stored database [24]. The Fourier transform recognizes the impurities and generates a report on the sample composition automatically [63].

7.3. Lack of Reference. In case of lack of reference (infrared of the known sample), an essential interpretation of spectrum of unknown sample could be effected from the first principles [90]. The first principles method is centered on the structural features of the molecule entailing the moieties attached to the molecules produce distinct and repeatable absorptions in the spectrum [90].

\section{Maintenance of Infrared Instrument}

Maintenance is essential for infrared spectrometer in order to obtain durability and availability when needed. Availability when needed is hindered in most cases because of not having a standby power stabilizer to prevent power failure. In addition, the instrument in most cases is uncovered. Covering them will prevent it from dust and, as well, ensure a good result.

\section{Concept of Infrared Spectroscopy-Based Synchrotron Radiation}

9.1. Infrared Using Synchrotron Radiation. Infrared based synchrotron radiation provides an efficient technique to characterize chemical compounds crosswise surfaces to determine the sample's chemical composition [93, 94]. Synchrotron radiation (SR) is the intense light produced by a synchrotron [95-97]. It covers a very wide electromagnetic spectrum from radio waves to infrared, visible light, ultraviolet light, $\mathrm{X}$-rays, and gamma rays [98]. Its distinct properties are intense brightness, broadband spectrum, polarization, and pulsed nature [99-101]. The intense brightness (brilliance) enables the detection of small quantities of various chemical compositions with a betterquality signal-to-noise ratio, whereas the extremely collimated light allows high-resolution special mapping of the chemical distribution [102-104].

9.2. Synchrotron Facility. A synchrotron is a basis for very bright (brilliant) light, which operates like a gigantic microscope to allow the matter to be seen at the atomic level $[105,106]$. Synchrotron light is a million times brighter than sunlight and a million times more intense than conventional 
X-rays [107]. According to Blaustein, synchrotron produces $\mathrm{X}$-ray which is a trillion times brighter than the conventional $\mathrm{X}$-rays [108]. Globally, there are over seventy synchrotrons $[109,110]$. Several of them are partly or fully devoted to synchrotron radiation generation for research purposes [110].

Synchrotron, which produces the synchrotron radiation (SR), is lay-down accessories referred to as synchrotron facility [108]. The accessories entail three main components, namely, a particle accelerator, series of magnets, and electric field [111]. The particle accelerators (linear and booster accelerators) accelerate charged particles (usually electrons) [112]. The series of magnets (focusing magnets, bending magnets, insertion devices (undulators), and wigglers) provide magnetic fields to rotate the particles in a circular path called storage ring [113]. The electric field accelerates the particles, and all the three main components are synchronized with the travelling particle beam $[114,115]$. A beamline is an experimental location, which uses a particular energy domain released by the synchrotron for research [116-118].

9.3. Infrared (IR) Microspectroscopy. Fourier Transform Infrared (FTIR) spectroscopy is a distinguished technique which probes the vibrational modes of molecules $[119,120]$. In this way, it provides a spectrum which is structure-specific (MFBU). The FTIR is used generally to study bulk homogeneous materials [121]. However, for the past ten years, the developments in infrared sources, optics, and detectors have led to the merging of FTIR spectroscopy with light spectroscopy to allow spectroscopic microscopy in complex environment of chemical compounds. In addition, since FTIR is nonionizing, compositional variations can be investigated as time goes by on time scales from a few milliseconds to hours. Infrared (IR) microspectroscopy, as implied in the name, is a combination of vibrational mid-infrared (IR) spectroscopy and microscopy $[23,122-124]$. It enables chemical composition of materials to be determined at microscopic level $[125,126]$. In general, an IR transmission microscope, with all reflecting optics is attached to a Fourier Transform IR (FTIR) spectrometer, allowing mid-IR absorption spectra to be collected from measurements as small as $5-10 \mu \mathrm{min}$, a sample to be analyzed [127]. This method depends on the use of a high numerical aperture (NA) condenser and objective optics to center the IR beam onto the sample, with ensuring beam collection which passed through the sample [121]. Optical diffraction limit at an analyzing wavelength is used to set the sample dimensions' limit to be studied [23]. Aperture insertion into the optical path of the IR beam at a central position paced either before or after the sample usually determines the selected region of interest for analysis [128]. This leads to a loss of most intense beam from reaching the detector in a conventional IR source, such as GlobarTM source [129]. On another note, multipixel IR imaging detectors, such as linear array and focal plane array (FPA) detectors, could be used to allow simultaneous collection of FT-IR spectra from various positions in the sample
$[23,130,131]$. However, this method usually depends on less than $0.1 \%$ of the IR source total intensity, being aimed at every sensing element of the FPA detector [23]. This other method merges the intense brightness of the wideband synchrotron source with an FT-IR spectrometer and IR microscope, which allows the full intensity of the IR source to be focused on a diffraction-limited position on the sample $[23,132]$. The outcome is a high signal-to-noise ratio (SNR) when the samples are analyzed with a magnitude of $5-10 \mu \mathrm{m}[133,134]$. A disadvantage associated with FTIR microspectroscopy (FTIRM) is the long wavelength of infrared light $[24,135,136]$. Here, the diffractionlimited spatial resolution is predicted theoretically as $2-10 \mu \mathrm{m}$ in the mid-infrared region $\left(4000-500 \mathrm{~cm}^{-1}\right)$ $[136,137]$. However, the brightness of the conventional thermal infrared source in laboratory FTIR spectrometers limits the spatial resolution $[130,136,138,139]$. At this very moment, synchrotron radiation plays an essential role because it is hundred to thousand times brighter than a conventional thermal source, therefore, enabling diffraction-limited spatial resolution to be attained [130, 136, 138, 139].

In summary, a synchrotron infrared source offers very much improved data which possess high signal-to-noise ratio and faster acquisition times for brightness-limited experiments, such as those involving high spatial or spectral resolution, or time-resolved measurements.

\section{Conclusion and Ongoing Research}

The relevance of infrared spectroscopy is worthwhile in order to achieve detailed result of the analyzed sample in research institutions and industries. This indicates that IR is a very versatile characterization technique. Distinct characteristics of intense brightness, high-resolution, and high signal-to-noise ratio (SNR) qualities of infrared based synchrotron radiation made it more excellent than FT-IR.

\section{Future Research}

At present, synchrotron IR beamlines committed to high spatial resolution analysis of microscopic materials are functioning in developed countries globally. It will be launched in South Africa (developing country) in 2020.

\section{Data Availability}

The data in the document and figures used to support the findings in this study are included within the research article.

\section{Conflicts of Interest}

The author declares no conflicts of interest.

\section{Acknowledgments}

The author appreciates Govan Mbeki Research and Development Centre (GMRDC) for financial assistance. 


\section{References}

[1] H. Uslu, D. Yankov, K. L. Wasewar, S. Azizian, N. Ullah, and W. Ahmad, "Separation of organic and inorganic compounds for specific applications," Journal of Chemistry, vol. 2015, Article ID 698259, 3 pages, 2015.

[2] R. Escobedo, R. Miranda, and J. Martínez, "Separation of organic and inorganic compounds for special applications," International Journal of Molecular Sciences, vol. 17, pp. 1-26, 2016.

[3] K. Arora and A. Parma, "Simulation of IR spectra of some organic compounds-A review," IOSR Journal of Applied Chemistry, vol. 6, no. 1, pp. 10-24, 2013.

[4] L. D'Souza, P. Devi, D. Shridhar, and C. G. Naik, "Use of fourier transform infrared (FTIR) spectroscopy to study cadmium-induced changes in Padina tetrastomatica," Analytical Chemistry Insights, vol. 3, pp. 135-143, 2008.

[5] A. E. Krauklis, A. I. Gagani, and A. T. Echtermeyer, "Near infrared spectroscopic method for monitoring water content in epoxy resins and fiber-reinforced composites," Materials, vol. 11, no. 4, pp. 1-14, 2018.

[6] F. Kondagula and K. Molt, "Infrared spectrometric purity control of organic liquids and water," CLEAN-Soil, Air, Water, vol. 37, no. 12, pp. 955-962, 2009.

[7] A. Kumar and V. Dangi, "Electromagnetic spectrum and its impact on human life," International Journal of All Research Education \& Scientific Methods, vol. 4, pp. 67-72, 2016.

[8] F. Tabrah, "Human injury from atomic particles and photon exposure: fears, myths, risks, and mortality," Hawai i Journal of Medicine, vol. 69, pp. 93-98, 2010.

[9] T. Wu, T. S. Rappaport, and C. M. Collins, "Safe for generations to come: considerations of safety for millimeter waves in wireless communications," IEEE Microwave Magazine, vol. 16, no. 2, pp. 65-84, 2015.

[10] A. Thielens, D. Bell, D. B. Mortimore, M. K. Greco, L. Martens, and W. Joseph, "Exposure of insects to radio frequency electromagnetic fields from 2 to $120 \mathrm{GH}$," Scientific Reports, vol. 8, pp. 1-10, 2018.

[11] F. Vatansever and M. R. Hamblin, "Far infrared radiation (FIR): its biological effects and medical applications," Photonics \& Lasers in Medicine, vol. 1, no. 4, pp. 255-266, 2012.

[12] S. Romanenko, R. S. Begley, A. R. Harvey, L. Hool, and V. P. Wallace, "The interaction between electromagnetic fields at megahertz, gigahertz, and terahertz frequencies with cells, tissues and organisms: risks and Potential," Journal of The Royal Society Interface, vol. 14, no. 137, pp. 1-22, 2017.

[13] I. Frau, O. Korostynska, A. Mason, and P. Byrne, "Comparison of electromagnetic wave sensors with optical and low-frequency spectroscopy methods for real-time monitoring of lead concentrations in mine water," Mine Water and the Environment, vol. 37, no. 3, pp. 617-634, 2018.

[14] M. Oziel, M. Hjouj, C. A. Gonzalez, J. Laveel, and B. Rubinsky, "Non-ionizing radio frequency electromagnetic waves traversing the head can be used to detect cerebrovascular autoregulation responses," Scientific Reports, vol. 6, no. 1, pp. 1-11, 2016.

[15] D. G. Sinex, "Spectral processing and sound source determination," International Review of Neurobiology, vol. 70, pp. 371-398, 2005.

[16] B. Hong, F. Monifi, and Y. Fainman, "Channel dispersed fourier transform spectrometer," Communications Physics, vol. 1, no. 1, pp. 1-8, 2018.

[17] V. Dang and F. Marini, "Chemometrics-based spectroscopy for pharmaceutical and biomedical analysis," Frontiers of Chemistry, vol. 7, pp. 1-2, 2019.
[18] A. Goto and S. Himeji, "Quantitative analysis of rock samples by an X-ray fluorescence spectrometer," Rigaku Journal, vol. 11, pp. 40-59, 1994

[19] S. Türker-Kaya and C. W. Huck, "A review of mid-infrared and near-infrared imaging: principles, concepts and applications in plant tissue analysis," Molecules, vol. 22, no. 1, pp. 1-20, 2017.

[20] N. Kalfaginnis, J. L. Stoner, J. Hiller, I. Vangelidis, and E. Lidorikis, "Mid-to-far-infrared sensing: $\mathrm{SrTiO}_{3}$, a novel optical material," Journal of Materials Chemistry, vol. 7, pp. 7851-7857, 2019.

[21] C. Pasquini, "Near infrared spectroscopy: fundamentals, practical aspects and analytical applications," Journal of the Brazilian Chemical Society, vol. 14, no. 2, pp. 198-219, 2003.

[22] G. Bellisola and C. Sorio, "Infrared spectroscopy and microscopy in cancer research and diagnosis," American Journal of Cancer Research, vol. 2, no. 1, pp. 1-21, 2012.

[23] R. Bhargava, "Infrared spectroscopic imaging: the next generation," Applied Spectroscopy, vol. 66, no. 10, pp. 1091-1120, 2012.

[24] M. J. Baker, J. Trevisan, P. Bassan et al., "Using fourier transform IR spectroscopy to analyze biological materials," Nature Protocols, vol. 9, no. 8, pp. 1771-1791, 2014.

[25] J. Booyens and M. P. Thantsha, "Transform infra-red spectroscopy and flow cytometric assessment of the antibacterial mechanism of action extract (Allium sativum) against selected Probiotic bifidobacterium strains," BMC Complementary and Alternative Medicine, vol. 14, no. 1, pp. 1-11, 2014.

[26] M. Smith, K. Thompson, and F. Lennard, "A literature review of analytical techniques for materials characterisation of painted textiles-Part 2: spectroscopic and chromatographic analytical instrumentation," Journal of the Institute of Conservation, vol. 40, no. 3, pp. 252-266, 2017.

[27] K. Hashimoto, V. R. Badarla, A. Kawai, and T. Ideguchi, "Complementary vibrational spectroscopy," Nature Communications, vol. 10, no. 1, pp. 1-6, 2019.

[28] A. Paterova, H. Yang, C. An, D. Kalashnikov, and L. Krivitsky, "Measurement of infrared optical constants with visible photons," New Journal of Physics, vol. 20, no. 4, pp. 1-11, 2018.

[29] M. Manzoli, "Boosting the characterization of heterogeneous catalysts for $\mathrm{H}_{2} \mathrm{O}_{2}$ direct synthesis by infrared spectroscopy," Catalysts, vol. 9, no. 1, pp. 1-34, 2019.

[30] R. Gautam, S. Vanga, F. Ariese, and S. Umapathy, "Review of multidimensional data processing approaches for Raman and infrared spectroscopy," EPJ Techniques and Instrumentation, vol. 2, no. 1, pp. 1-38, 2015.

[31] S. Moon, S.-W. Lee, and Z. Chen, "Reference spectrum extraction and fixed-pattern noise removal in optical coherence tomography," Optics Express, vol. 18, no. 24, 2010.

[32] M. Almeida, K. E. Torrance, and A. K. Datta, "Measurement of optical properties of foods in near- and mid-infrared radiation," International Journal of Food Properties, vol. 9, no. 4, pp. 651-664, 2006.

[33] A. C. Ceccacci, A. Cagliani, P. Marizza, S. Schmid, and A. Boisen, "Thin film analysis by nanomechanical infrared spectroscopy," ACS Omega, vol. 4, pp. 7626-7635, 2019.

[34] F. Long, Z. Chen, K. Han, L. Zhang, and W. Zhuang, "Differentiation between enamines and tautomerizable imines oxidation reaction mechanism using electron-vibrationvibration two dimensional infrared spectroscopy," Molecules, vol. 24, no. 5, pp. 1-15, 2019. 
[35] A. L. Le Sueur, R. E. Horness, and M. C. Thielges, "Applications of two-dimensional infrared spectroscopy," The Analyst, vol. 140, no. 13, pp. 4336-4349, 2015.

[36] F. Cheng, X. Yang, and J. Gao, "Ultrasensitive detection and characterization of molecules with infrared Plasmonic metamaterials," Scientific Reports, vol. 5, no. 1, pp. 1-10, 2015.

[37] G. ElMasry and S. Nakauchi, "Prediction of meat spectral patterns based on optical properties and concentrations of the major constituents," Food Science \& Nutrition, vol. 4, no. 2, pp. 269-283, 2016.

[38] M. Munajad, C. Subroto, and Suwarno, "Fourier transform infrared (FTIR) spectroscopy analysis of transformer paper in mineral oil-paper composite insulation under accelerated thermal aging," Energies, vol. 11, no. 2, pp. 1-12, 2018.

[39] F. Y. Sun, D. Hou, Q. S. Bai, and X. H. Huang, "Rabi resonance in $\mathrm{Cs}$ atoms and its application to microwave magnetic field measurement," Journal of Physics Communications, vol. 2, no. 1, pp. 1-10, 2018.

[40] J. D. Kubicki and H. D. Watts, "Quantum mechanical modeling of the vibrational spectral of minerals with a focus on clays," Minerals, vol. 9, no. 3, pp. 1-20, 2019.

[41] B. Xianga, R. F. Ribeiro, A. D. Dunkelberger et al., "Twodimensional infrared spectroscopy of vibrational Polaritons," Proceedings of the National Academy of Sciences, vol. 10, pp. 1-6, 2017.

[42] T. A. A. Oliver, N. H. C. Lewis, and G. R. Fleming, "Correlating the motion of electrons with two-dimensional electronic-vibrational spectroscopy," Proceedings of the National Academy of Sciences, vol. 4, no. 111, pp. 1006110066, 2017.

[43] K. Salazar-Salinas, P. A. Baldera-Aguayo, J. J. EncomenderoRisco et al., "Metal-ion effects on the polarization of metalbound water an infrared vibrational modes of the coordinated metal center of Mycobacterium tuberculosis pyrazinamidase via quantum mechanical calculations," The Journal of Physical Chemistry B, vol. 118, no. 34, pp. 100065-110075, 2014.

[44] R. Garg, "Fourier transform infrared spectroscopic characterization for detecting soil minerals and toxicological effects of selected minerals in soils," Acta Chimica and Pharmaceutica Indica, vol. 2, pp. 242-253, 2012.

[45] K. L. Chubb, A. Yachmenev, J. Tennyson, and S. N. Yurchenko, "Treating linear molecule $\mathrm{HCCH}$ in calculations of rotation-vibration spectra," The Journal of Chemical Physics, vol. 149, no. 1, pp. 1-18, 2018.

[46] A. Zen, D. Zhelyazov, and L. Guidoni, "Optimized structure and vibrational properties by error affected potential energy surfaces," Journal of Chemical Theory and Computation, vol. 8, no. 11, pp. 4204-4215, 2012.

[47] M. Subr and M. Prochazka, "Polarization and angular-resolved optical response of molecules on anisotropic plasmonic nanostructures," Nanomaterial, vol. 8, no. 6, pp. 1-38, 2018.

[48] G. Mahalakshmi and V. Balachandran, "Molecular structure, vibrational spectra (FTIR and FT Raman) and natural bond orbital analysis of 4-Aminomethylpiperidine: DFT study," Spectrochimica Acta Part A: Molecular and Biomolecular Spectroscopy, vol. 131, pp. 587-596, 2014.

[49] K. Wang, Y. Yuan, S. Han, and Y. Yang, "Application of FTIR spectroscopy with solvent-cast film and PLS regression for the quantification of SBS content in modified asphalt," International Journal of Pavement Engineering, vol. 20, no. 11, pp. 1336-1341, 2019.
[50] C. Hernández-Aguilar, A. Domínguez-Pacheco, A. CruzOrea, and R. Ivanov, "Photoacoustic spectroscopy in the optical characterization of foodstuff: a review," Journal of Spectroscopy, vol. 2019, Article ID 5920948, 34 pages, 2019.

[51] L. Bokobza, "Spectroscopic techniques for the characterization of polymer nanocomposites," Polymers, vol. 10, no. 1, pp. 1-21, 2017.

[52] V. Bellon, J. L. Vigneau, and F. Sévila, "Infrared and nearinfrared technology for the food industry and agricultural uses: on-line applications," Food Control, vol. 5, no. 1, pp. 21-27, 1994.

[53] M. Sega, D. M. E. Amico, and M. Plassa, "Stability of a NDIR analyser for $\mathrm{CO}_{2}$ at atmospheric concentration," Annali di Chimica, vol. 92, no. 9, pp. 897-902, 2002.

[54] M. A. Abbas, Q. Pan, J. Mandon, S. M. Cristescu, F. J. M. Harren, and A. Kodabakhsh, "Time-resolved midinfrared dualcomb spectroscopy," Scientific Reports, vol. 9, no. 1, pp. 1-9, 2019.

[55] R. A. Crocombe, "Portable spectroscopy," Applied Spectroscopy, vol. 72, no. 12, pp. 1701-1751, 2018.

[56] L. Wang, H. Wang, M. Wagner, Y. Yan, D. S. Jacob, and X. G. Xu, "Nanoscale simultaneous chemical and mechanical imaging via peak force infrared microscopy," Science Advances, vol. 3, no. 6, pp. 1-12, 2017.

[57] R. Usamentiaga, P. Venegas, J. Guerediaga, L. Vega, J. Molleda, and F. Bulnes, "Infrared thermography for temperature measurement and non-destructive testing," Sensors, vol. 14, no. 7, pp. 12305-12348, 2014.

[58] N. Ahmed, C. B. Singh, S. Bhattacharya, S. Dhara, and P. B. Bhargav, "Raman and FTIR studies on PECVD grown ammonia-free amorphous silicon nitride thin films for solar cell applications," Conference Papers in Energy, vol. 2013, Article ID 837676, 4 pages, 2013.

[59] V. N. Bessolov, Y. V. Zhikyaev, E. N. Konenkova, V. A. Fedirko, and D. R. T. Zahn, "Raman and infrared spectroscopy of $\mathrm{GaN}$ nanocrystals grown by chloro-hydride vapour-Phase epitaxy on oxidized silicon," Semiconductors, vol. 37, no. 8, pp. 640-643, 2003.

[60] S. S. Harilal, B. E. Brumfield, N. I. LaHaye, K. C. Hartig, and M. C. Phillips, "Optical spectroscopy of laser-produced Plasmas for standoff isotopic analysis," Applied Physics Reviews, vol. 5, no. 2, pp. 1-33, 2018.

[61] L. He, Q. Zhai, P. Lan et al., "Monitoring ultrafast vibrational dynamics of isotopic molecules with frequency modulation of high-order harmonics," Nature Communications, vol. 9, no. 1, pp. 1-7, 2018.

[62] S. A. Krillov, "Novel approaches in spectroscopy of interparticle inter-actions. Vibrational line Profiles and anomalous non-coincidence effects," Novel Approaches to the Structure and Dynamics of Liquids: Experiments, Theories and Simulations, Springer, Berlin, Germany, pp. 193-227, 2004.

[63] L. Bokobza, "Some applications of vibrational spectroscopy for the analysis of polymers and polymer composites," Polymers, vol. 11, no. 7, pp. 1-13, 2019.

[64] T. La C. Jansen, S. Saito, J. Jeon, and M. Co, "Theory of coherent two-dimensional vibrational spectroscopy," The Journal of Chemical Physics, vol. 150, no. 10, pp. 1-18, 2019.

[65] K. B. Beć and C. W. Huck, "Breakthrough potential in nearinfrared spectroscopy: spectral simulation. A review of recent developments," Frontiers of Chemistry, vol. 7, pp. 1-22, 2019.

[66] L. Ma, Y. Peng, Y. Pei et al., "Systematic discovery about NIR spectral assignment from chemical structural property to 
natural chemical compounds," Scientific Reports, vol. 9, no. 1, pp. 1-18, 2019.

[67] J. S. Ostrander, A. L. Serrano, A. Ghosh, and M. T. Zanni, "Spatially resolved two-dimensional infrared spectroscopy via wide-field microscopy," ACS Photonics, vol. 3, no. 7, pp. 1315-1323, 2016.

[68] S. S. Mukherjee, D. R. Skoff, C. T. Middleton, and M. T. Zannia, "Fully absorptive 3D IR spectroscopy using a dual mid-infrared pulse sharper," The Journal of Chemical Physics, vol. 139, no. 14, pp. 1-10, 2013.

[69] M. T. Zanni, "Two-dimensional infrared spectroscopy measures the structural dynamics of a self-assembled film only one molecule thick," Proceedings of the National Academy of Sciences, vol. 113, no. 18, pp. 4890-4891, 2016.

[70] T. Fuji, H. Shirai, and Y. Nomura, "Development and application of sub-cycle mid-infrared source based on laser filamentation," Applied Science, vol. 7, pp. 1-5, 2017.

[71] T. A. A. Oliver, "Recent advances in multidimensional ultrafast spectroscopy," Royal Society Open Science, vol. 5, no. 1, pp. 1-21, 2018.

[72] M. Woerner, W. Kuehn, P. Bowlan, K. Reimann, and T. Elsaesser, "Ultrafast two-dimensional terahertz spectroscopy of elementary excitations in solids," New Journal of Physics, vol. 15, no. 2, pp. 1-17, 2013.

[73] R. Borreo-Varillas, A. Nenov, L. Ganzer et al., "Two-dimensional UV spectroscopy: a new insight into the structure and dynamics of biomolecules," Chemical Science, vol. 10, pp. 1-15, 2019.

[74] P. Salén, M. Basini, S. Bonetti et al., "Matter manipulation with extreme terahertz light: progress in the enabling $\mathrm{THz}$ technology," Physics Reports, vol. 836-837, pp. 1-74, 2019.

[75] R. Geneaux, H. J. B. Marroux, A. Guggenmos, D. M. Neumgark, and S. R. Leone, "Transient absorption spectroscopy using harmonic generation: a review of ultrafast X-ray dynamics in molecules and solids," Philosophical Transactions of the Royal Society A: Mathematical, Physical and Engineering Sciences, vol. 377, no. 2145, pp. 127, 2019.

[76] V. V. Krishnan, "Molecular thermodynamics using nuclear magnetic resonance (NMR) spectroscopy," Inventions, vol. 4, no. 1, pp. 1-19, 2019.

[77] M. Ohmehaeuser, Y. B. Monakhova, T. Kuballa, and D. W. Lachenmeier, "Qualitative and quantitative control of honeys using NMR spectroscopy and chemometrics," ISRN Analy. Chem.vol. 2013, Article ID 825318, 9 pages, 2013.

[78] W. Xiong, J. E. Laaser, R. D. Mehlenbacher, and M. T. Zanni, "Adding a dimension to the infrared spectra of interfaces using heterodyne detected 2D sum-frequency generation (HD 2D SFG) spectroscopy," Proceedings of the National Academy of Sciences, vol. 108, no. 52, pp. 20902-20907, 2011.

[79] F. Lon, Z. Cen, K. Han, L. Zhang, and W. Zhuang, "Differentiation between enamines and tautomerizable imines oxidation reaction mechanism using electron-vibration-vibration two dimensional infrared spectroscopy," Molecules, vol. 24, no. 5, pp. 1-15, 2019.

[80] R. C. Breton and W. F. Reynolds, "Using NMR to identify and characterize natural products," Natural Product Reports, vol. 30, no. 4, pp. 501-524, 2013.

[81] G. F. Pauli, M. Niemitz, J. Bisson et al., "Toward structural correctness: aquatolide and the importance of 1D Proton NMR FID archiving," The Journal of Organic Chemistry, vol. 81, no. 3, pp. 878-889, 2016.
[82] D. Marion, "An introduction to biological NMR spectroscopy," Molecular \& Cellular Proteomics, vol. 12, no. 11, pp. 3006-3025, 2013.

[83] P. C. Chen, "An introduction to coherent multidimensional spectroscopy," Applied Spectroscopy, vol. 70, no. 12, pp. 1937-1951, 2016.

[84] B. Xiang, R. F. Ribeiro, A. D. Dunkelberger et al., "Twodimensional infrared spectroscopy of vibrational Polaritons," Proceedings of the National Academy of Sciences, vol. 115, no. 19, pp. 4845-4850, 2018.

[85] J. S. Ostrander, J. P. Lomont, K. L. Rich et al., "Monolayer sensitivity enables a 2D IR spectroscopic immuno-biosensor for studying protein structures: application to amyloid Polymorphs," The Journal of Physical Chemistry Letters, vol. 10, no. 14, pp. 3836-3842, 2019.

[86] L. V. Resende and C. C. Nascentes, "A simple method for the multi-elemental analysis of organic fertilizer by slurry sampling and total reflection X-ray fluorescence," Talanta, vol. 147, pp. 485-492, 2016

[87] O. Rahmani, M. Khoshnoodkia, A. Kadkhodaie, B. A. Pour, and H. Tsegab, "Geochemical analysis for determining total organic carbon content based on $\Delta \operatorname{LogR}$ technique in the South Pars field," Minerals, vol. 9, no. 12, pp. 1-13, 2019.

[88] M. R. Siddiqui, Z. A. AlOthman, and N. Rahman, "Analytical techniques in pharmaceutical analysis: a review," Arabian Journal of Chemistry, vol. 10, pp. S1409-S1421, 2017.

[89] D. F. Barbin, A. L. d. S. M. Felicio, D.-W. Sun, S. L. Nixdorf, and E. Y. Hirooka, "Application of infrared spectral techniques on quality and compositional attributes of coffee: an overview," Food Research International, vol. 61, pp. 23-32, 2014.

[90] J. Coates, "Interpretation of infrared spectra, a practical approach,"'Interpretation of infrared spectra, a practical approach," in Encyclopedia of Analytical Chemistry, R. A. Meyer, Ed., pp. 117-124, John Wiley \& Sons, Hoboken, NJ, USA, 2000.

[91] L. Harper, J. Powell, and E. M. Pijl, “An overview of forensic drug testing methods and their suitability for harm reduction point-of-care services," Harm Reduction Journal, vol. 14, no. 1, pp. 1-13, 2017.

[92] W.-H. Su and D.-W. Sun, "Fourier transform infrared and Raman and hyperspectral imaging techniques for quality determinations of Powdery foods: a review," Comprehensive Reviews in Food Science and Food Safety, vol. 17, no. 1, pp. 104-122, 2018.

[93] J. Vongsviviut, V. K. Truong, M. A. Kobaisi et al., "Synchrotron macro ATR-FTIR microspectroscopic analysis of silica nanoparticles-embedded Polyester steel surfaces subjected to Prolonged UV and humidity exposure," PLoS One, vol. 12, no. 12, pp. 1-16, 2017.

[94] S. M. Oliveira Junior, M. F. Diniz, R. C. L. Dutra, M. Massi, and C. Otani, "Applications of FT-IR techniques and goniometry on characterization of carbon fiber surfaces," Journal of Aerospace Technology and Management, vol. 8, pp. 26-32, 2016.

[95] N. Gazis, E. Tanke, T. Apostolopoulos, K. Pramatari, R. Rochow, and E. Gazis, "Light sources in Europe-case study: the COMPACTLIGHT collaboration," Instruments, vol. 3, no. 3, pp. 1-11, 2019.

[96] S. D. Mitri, "One way to synchrotron light sources upgrade?" Journal of Synchrotron Radiation, vol. 25, pp. 1323-1334, 2018. 
[97] M. Chen, J. Luo, F. Li, F. Liu, Z. Shen, and J. Zhang, "Tunable synchrotron-like radiation from centimeter scale plasmas channels," Light: Science \& Applications, vol. 5, pp. 1-7, 2018.

[98] L. M. Miller and P. Dumas, "Infrared spectroscopy using synchrotron radiation," "Infrared spectroscopy using synchrotron radiation," Edited by G. Roberts and A. Watts, Eds., Springer, Berlin, Germany, 2018.

[99] J. Luo, M. Chen, M. Zeng et al., "A compact tunable polarized $\mathrm{X}$-ray source based on laser-plasma helical undulators," Scientific Reports, vol. 6, no. 1, pp. 1-8, 2016.

[100] A. Döpp, B. Mahieu, A. Lifschitz et al., "Stable femtosecond $\mathrm{X}$-rays with tunable polarization from a laser-driven accelerator," Light: Science \& Applications, vol. 6, pp. 1-7, 2017.

[101] C. Gaida, M. Gebhardt, T. Heuermann et al., "Watt-scale super-octave mid-infrared intrapulse difference frequency generation," Light: Science \& Applications, vol. 7, pp. 1-8, 2018.

[102] H. Chen, C. Lu, J. Liu et al., "Synchrotron radiation X-ray powder diffraction techniques applied in hydrogen storage materials-a review," Progress in Natural Science: Materials Internationa, vol. 77, pp. 66-73, 2017.

[103] F. Meirer and B. M. Weckhuysen, "Spatial and temporal exploration of heterogeneous catalysts with synchrotron radiation," Nature Reviews Materials, vol. 3, no. 9, pp. 324-340, 2018.

[104] M. R. Gherase and D. E. B. Fleming, "Probing trace elements in human tissues wit synchrotron radiation," Crystals, vol. 10, no. 1, pp. 1-24, 2020.

[105] J. Rossbach, J. R. Schneider, and W. Wurth, "10 Years of Pioneering X-ray science at the free-electron laser FLASH at DESY," Physics Reports, vol. 808, pp. 1-74, 2019.

[106] V. Weinhardt, J.-H. Chen, A. Ekman, G. McDermott, M. A. Le Gros, and C. Larabell, "Imaging cell morphology and physiology using X-rays," Biochemical Society Transactions, vol. 47, no. 2, pp. 489-508, 2019.

[107] G. W. Rhoades, G. S. Belev, L. D. Chapman et al., "Diffraction-enhanced computed tomographic Imaging of growing Piglet joints by using a synchrotron light source," Comparative Medicine, vol. 65, no. 4, pp. 342-347, 2015.

[108] R. Blaustein, "Biology and light sources: synchrotrons allow researchers a deep look into life," BioScience, vol. 67, no. 8, pp. 201-207, 2017.

[109] P. Suwanpinij, "The synchrotron radiation for steel research," Advances in Materials Science and Engineering, vol. 2016, Article ID 2479345, 6 pages, 2016.

[110] V. Simoulsin, "The synchrotron generations: communities and facilities at the crossroads between the national and international," French Journal of Sociology, vol. 57, no. 3, pp. 503-528, 2016.

[111] P. K. Dey, "Power supplies for high energy particle accelerators," Journal of the Institution of Engineers (India): Series B, vol. 97, no. 2, pp. 253-267, 2016.

[112] H. Wiedermann, "Introduction to accelerator physics," in Particle Accelerator Physics, pp. 3-41, Springer, Berlin, Germany, 2015.

[113] H. Wiedermann, "Insertion device radiation," in Particle Accelerator Physics, pp. 895-927, Springer, Berlin, Germany, 2015.

[114] H. Wiedermann, "Longitudinal beam dynamics," in Particle Accelerator Physics, pp. 253-302, Springer, Berlin, Germany, 2015.

[115] S. Myers, "The engineering needed for particle Physics," Philosophical Transactions of the Royal Society A:
Mathematical, Physical and Engineering Sciences, vol. 370, no. 1973, pp. 3887-2923, 2012.

[116] L. Rebuffi, M. Sánchez del Río, and M. S. ShadowQui, "ShadowOui: a new visual environment for X-ray optics and synchrotron beamline simulations," Journal of Synchrotron Radiation, vol. 23, no. 6, pp. 1357-1367, 2016.

[117] S. Pascarelli, O. Mathon, T. Mairs et al., "The Time-resolved and Extreme-conditions XAS (TEXAS) facility at the European synchrotron radiation facility: the energy-dispersive X-ray absorption spectroscopy beamline ID24," Journal of Synchrotron Radiation, vol. 23, no. 1, pp. 353-368, 2016.

[118] N. Hirao, S. I. Kawaguchi, I. K. Hirose, K. Shimizy, E. Ohtani, and Y. Ohishi, "New developments in high-pressure X-ray diffraction beamline for diamond anvil cell at spring-8," Matter and Radiation at Extremes, vol. 5, pp. 1-10, 2020.

[119] L. M. Miller and P. Dumas, "From structure to cellular mechanism with infrared microspectroscopy," Current Opinion in Structural Biology, vol. 20, no. 5, pp. 649-656, 2010.

[120] C. Karunakaran, C. R. Christensen, C. Galliard et al., "Introduction of soft X-ray spectromicroscopy as an advanced technique for plant biopolymers research," PLoS One, vol. 10, no. 3, pp. 1-15, 2015.

[121] Y. Chen, C. Zou, M. Mastalerz, S. Hu, C. Gasaway, and $\mathrm{X}$. Tao, "Applications of micro-fourier transform infrared spectroscopy (FTIR) in the geological sciences-A review," International Journal of Molecular Sciences, vol. 16, no. 12, pp. 1-28, 2015.

[122] T. P. Wrobel and R. Bhargava, "Infrared spectroscopic imaging advances as an analytical technology for biomedical sciences," Analytical Chemistry, vol. 90, no. 3, pp. 1444-1463, 2018.

[123] F. Faghihzadeh, N. M. Anaya, L. A. Schiffman, and V. Oyandel-Craver, "Fourier transform infrared spectroscopy to assess molecular-level changes in microorganisms exposed to nanoparticles," Nanotechnology for Environmental Engineering, vol. 1, no. 1, pp. 1-16, 2016.

[124] V. Balan, C. Mhai, F. Cojocaru, C. Uritu, B. Dodi, and I. Gardikiotis, "Vibrational spectroscopy fingerprinting in medicine: from molecular to clinical practice," Materials, vol. 12, no. 18, pp. 1-40, 2019.

[125] J. Vongsvivut, D. Pérez-Guaita, B. R. Wood et al., "Synchrotron macro ATR-FTIR microspectroscopy for highresolution chemical mapping of single cells," The Analyst, vol. 144, no. 10, pp. 3226-3238, 2019.

[126] J. Depciuch, I. Kasprzyk, E. Drzymała, and M. ParlinskaWojtan, "Identification of birch Pollen species using FTIR spectroscopy," Aerobiologia, vol. 34, no. 4, pp. 525-538, 2018.

[127] S. Primpke, M. Wirth, C. Lorenz, G. Gerdts, and G. Gerdts, "Reference database design for the automated analysis of microplastic samples based on fourier transform infrared (FTIR) spectroscopy," Analytical and Bioanalytical Chemistry, vol. 410, no. 21, pp. 5131-5141, 2018.

[128] H.-Y. N. Holman, H. A. Bechtel, Z. Hao, and M. C. Martin, "Synchrotron IR spectromicroscopy: chemistry of living cells," Analytical Chemistry, vol. 82, no. 21, pp. 8757-8765, 2010.

[129] G. J. Ellis and M. C. Martin, "Opportunities and challenges for polymer science using synchrotron-based infrared spectroscopy," European Polymer Journal, vol. 81, pp. 1-31, 2016.

[130] E. Stavitski, R. J. Smith, M. W. Bourassa, A. S. Acerbo, G. L. Carr, and L. M. Miller, "Dynamic full-field infrared 
imaging with multiple synchrotron beams," Analytical Chemistry, vol. 85, no. 7, pp. 3599-3605, 2013.

[131] C. J. Hirschmugl and K. M. Gough, "Fourier transform infrared spectrochemical imaging: review of design and applications with a focal plane array and multiple beam synchrotron radiation source," Applied Spectroscopy, vol. 66, no. 5, pp. 475-491, 2012.

[132] D. T. D. Childs, R. A. Hogg, D. G. Revin, I. U. Rehman, J. W. Cockburn, and S. J. Matcher, "Sensitivity advantage of QCL tunable-laser mid-infrared spectroscopy over FTIR spectroscopy," Applied Spectroscopy Reviews, vol. 50, no. 10, pp. 822-839, 2015.

[133] Y. Tseng, P. Bouzy, C. Pedersen, N. Stone, and P. TidemandLichtenberg, "Upconversion raster scanning microscope for long-wavelength infrared imaging of breast cancer microcalcifications," Biomedical Optics Express, vol. 9, pp. 1-9, 2018.

[134] M. Pilling and P. Gardner, "Fundamental developments in infrared spectroscopic imaging for biomedical applications," Chemical Society Reviews, vol. 45, no. 7, pp. 1935-1957, 2016.

[135] H. Wang, J. Peng, C. Xie, Y. Bao, and Y. He, "Fruit quality evaluation using spectroscopy technology: a review," Sensors, vol. 15, no. 5, pp. 11889-11927, 2015.

[136] H. A. Bechter, E. A. Muller, R. L. Olmon, M. C. Martin, and M. B. Raschke, "Ultrabroadband infrared nanospectroscopic imaging," Proceedings of the National Academy of Sciences, vol. 111, pp. 7191-7196, 2014.

[137] S. Simona, C. Carla, O. Giulia, and G. Elisabetta, "Infrared spectroscopy as a new tool for studying single living cells: is there a niche?" Biomedical Spectroscopy and Imaging, vol. 6, pp. 85-99, 2017.

[138] H.-Y. N. Holman, M. C. Martin, and W. R. McKinney, "Tracking chemical changes in a live cell: biomedical applications of SR-FTIR spectromicroscopy," Spectroscopy, vol. 17, no. 2-3, pp. 139-159, 2003.

[139] K. H. Michaelian, M. D. Frogley, G. Cinque, and L. Quaroni, "Infrared spectra of micro-structured samples with MicroPhotoacoustic spectroscopy and synchrotron radiation," The Analyst, vol. 145, no. 4, pp. 1483-1490, 2020. 